\title{
Tumor location is an independent predictive factor for distant metastasis and metastatic sites of rectal adenocarcinoma in patients receiving total mesorectal excision
}

\author{
Chien-Hsin Chen ${ }^{1}$, Mao-Chih Hsieh², Ping-Kun Hsiao ${ }^{2}$, En-Kwang Lin¹, Yen-Jung Lu ${ }^{1}$, Szu-Yuan Wu ${ }^{3,4,5,6 \bowtie}$ \\ 1. Department of Colorectal Surgery, Wan Fang Hospital, Taipei Medical University, Taipei, Taiwan \\ 2. Department of General Surgery, Wan Fang Hospital, Taipei Medical University, Taipei, Taiwan \\ 3. Institute of Toxicology, College of Medicine, NationalTaiwanUniversity, Taipei, Taiwan \\ 4. Department of Radiation Oncology, Wan Fang Hospital, Taipei Medical University, Taipei, Taiwan \\ 5. Department of Internal Medicine, School of Medicine, College of Medicine, Taipei Medical University, Taipei, Taiwan \\ 6. Department of Biotechnology, Hungkuang University, Taichung, Taiwan
}

$\square$ Corresponding author: Szu-Yuan Wu, M.D., M.P.H., Ph.D., Attending Physician, Department of Radiation Oncology, Taipei Medical University - Wan Fang Medical Center, NO.111, Section 3, Hsing-Long Rd, Taipei 116, Taiwan, R.O.C. E-mail: szuyuanwu5399@gmail.com

(c) Ivyspring International Publisher. This is an open access article distributed under the terms of the Creative Commons Attribution (CC BY-NC) license (https:// creativecommons.org/licenses/by-nc/4.0/). See http://ivyspring.com/terms for full terms and conditions.

Received: 2017.05.27; Accepted: 2018.02.20; Published: 2018.02.28

\begin{abstract}
Background and Objectives: To evaluate the predictive factor for and patterns of distant metastasis in patients with rectal adenocarcinoma receiving total mesorectal excision (TME).

Methods: We enrolled 217 consecutive patients who had histologically confirmed rectal adenocarcinoma and underwent surgery at Taipei Medical University- Wanfang Hospital between January 2000 and December 2014. TME was performed in all patients undergoing a sphincter-sparing procedure or abdominal perineal resection of rectal cancer. We performed univariate and multivariate Cox regression analyses of the distant metastasis rate in all patients to evaluate predictive factors. Overall survival (OS) rates were calculated using the Kaplan-Meier method, and Kaplan-Meier survival curves were compared using the log-rank test.

Results: A multivariate Cox regression analysis of the distant metastasis rate in patients with rectal adenocarcinoma identified tumor locations and American Joint Committee on Cancer (AJCC) stages as prognostic risk factors. The adjusted hazard ratios (aHRs) of distant metastasis for the upper-third, middle-third, and AJCC stage I-II cancers were $0.08(95 \% \mathrm{Cl}, 0.01-0.69 ; p=0.021), 0.41(95 \% \mathrm{Cl}$, $0.15-0.99 ; p=0.047)$, and $0.20(95 \% \mathrm{Cl}, 0.10-0.66 ; p=0.008)$, respectively. The 5 -year lung metastasis rates among patients with upper-, middle-, and lower-third rectal cancers were $0 \%, 3.37 \%$, and $13.33 \%$, respectively (log-rank, $p=0.001$ ), and the 5 -year liver metastasis rates among patients with upper-, middle-, and lower-third rectal cancers were $2.12 \%, 9.10 \%$, and $11.76 \%$, respectively (log-rank, $p=0.096$ ). The 5-year OS rates also differed with different rectal adenocarcinoma locations. The 5-year OS rates for upper, middle, and lower rectal cancers were 96\%, 86\%, and 64\%, respectively (log-rank, $p<0.001$ ).

Conclusion: A poor OS rate and high lung or liver metastasis rate were observed in distal rectal adenocarcinoma. Longer intensive surveillance of the chest, abdomen, and pelvis after TME in distal rectal adenocarcinoma could be necessary.
\end{abstract}

Key words: Rectal adenocarcinoma; total mesorectal excision; distant metastasis

\section{Introduction}

Approximately 4256 Taiwanese people are diagnosed as having rectal cancer annually.[1]
Adenocarcinomas constitute the vast majority of these cancers.[1] Primary rectal squamous cell carcinomas, 
which are very rare, can be difficult to distinguish from anal cancers and are treated using the same approach as that for anal cancer, with initial chemoradiotherapy (CCRT) rather than surgery.[2] The optimal approach to treating rectal adenocarcinoma depends upon numerous factors, of which the location in the rectum and the local disease extent are most important.[3] The treatment of distal rectal cancer is still a challenge in terms of reducing permanent stoma, reducing local recurrence, and improving survival.[3] The improvements in the surgical technique total mesorectal excision (TME), described by Healed et al. in 1982,[4-6] have resulted in a local recurrence rate of $4 \%-10 \%$. $[3-5,7,8]$

Although local recurrence appeared to considerably decrease because of the TME technique, the 5-year overall survival (OS) stratified by tumor stage at diagnosis for rectal cancer by using the 2010 staging criteria remains less than $50 \%$ in advanced stages.[9] Understanding the failure patterns of distant metastasis is very important, in addition to local recurrence in the era of TME. However, no strong predictive factor for distant metastasis exists, and the existing predictive factors have been used for determining outcomes of OS or local recurrence rather than those of distant metastasis.[9-12] Moreover, these predictive factors have been used for determining colon and rectal cancers, but are not specific for rectal adenocarcinoma.[9-12]

In the current study, our objective was to evaluate the predictive factors for distant metastasis in patients with rectal adenocarcinoma receiving TME. The patterns of distant metastasis including metastatic sites and metastatic interval were estimated in our study. Moreover, we evaluated whether the predictive factors for distant metastasis could be used for estimating the OS.

\section{Patients and Methods}

\section{Study Patients}

We enrolled 217 consecutive patients who had histologically confirmed rectal adenocarcinoma and underwent surgery at Taipei Medical UniversityWanfang Hospital between January 2000 and December 2014. All enrolled patients were Taiwanese (Asian population). After rectal surgery, the mean number of total harvested lymph nodes was 18.3 (standard deviation [SD], 9.6). The mean follow-up period was 80 months (SD, 37 months). Clinical and pathological data were reviewed to evaluate prognostic factors for the distant metastasis of rectal adenocarcinoma. Adjuvant radiotherapy (RT) and chemotherapy (CT) are indicated for pT3, pT4, or lymph node-positive rectal cancers in our hospital.[3]
Upper-, middle-, and lower-third rectal adenocarcinomas were defined as tumor margins $11.1-15 \mathrm{~cm}$ from, 7.1-11 cm from, and within $7 \mathrm{~cm}$ of the anal verge, respectively, as measured through rigid sigmoidoscopy.[13] Our protocols were reviewed and approved by the institutional review board of our hospital.

\section{Surgery and Follow-Up}

TME was performed in all patients undergoing a sphincter-sparing procedure or an abdominal perineal resection (APR) of rectal cancer. TME included high ligation of the inferior mesentery artery and vein; mobilization of the sigmoid colon, descending colon, or splenic flexure; and mobilization of the rectum through sharp dissection with diathermy or scissors under direct vision in the avascular plane between the visceral fascia of the mesorectum and the parietal fascia of the pelvis, as described by Heald et al.[5]

Pathological staging of the disease was performed according to the American Joint Committee on Cancer (AJCC) Cancer Staging Manual, 6th edition. After the surgery, all patients were enrolled in a surveillance program designed to detect disease status including local recurrence or distant metastasis. Clinic visits were scheduled every 3 months for the first 2 years and then at 6-month intervals for 3 years. During each visit, pelvic examination was performed, and the carcinoembryonic antigen level was measured. Abdominal ultrasound or computed tomography was performed every 6 months. Colonoscopy was performed after 1 and 3 years. If the patients missed the follow-up session at our outpatient department, we contacted them by telephone or mail. Any symptom potentially related to local tumor recurrence or distant metastasis was investigated through digital rectal examination, colonoscopy, and computed tomography or magnetic resonance imaging. Distant metastasis was confirmed through biopsy.

\section{Statistical Analysis}

The primary endpoint of the study was confirmation of distant metastasis. Patients lost to follow-up were censored from the time of last follow-up. Patients with confirmed distant metastasis (confirmed through pathological findings) were compared with those without confirmed distant metastasis. Continuous variables (expressed as medians (ranges)) were compared using the MannWhitney $U$ test or analysis of variance (ANOVA; two or more independent groups), whereas categorical variables (percentages) were compared using the chi-squared test or Fisher exact test, when indicated. Multivariate analysis was performed using Cox 
regression analysis for long-term follow-up (different time, censored data), and the analysis included only model variables having the highest or lowest $(p<0.05)$ univariate risk. Statistical significance was defined as $p<0.05$. Results are presented with a hazard ratio (HR) and 95\% confidence interval (CI). All $p$ values were two-tailed. Significant independent predictors for distant metastasis such as pathologic AJCC stages and locations of the rectum were determined using a multivariate Cox regression analysis to determine the $\mathrm{HR}$; the independent predictors were controlled for or stratified in the analysis, and the endpoint was the distant metastasis rate among the rectal adenocarcinoma locations. The cumulative proportion of the distant metastasis rate was calculated using the Kaplan-Meier method. Kaplan-Meier survival curves were compared using the log-rank test. Statistical analyses were performed using SPSS, Version 13.0, for Windows (SPSS Inc., Chicago, IL, USA).

\section{Results}

We enrolled 217 patients with rectal adenocarcinoma status post-surgery. The characteristics of these patients with and without distant metastasis following surgery are presented in Table 1 . Of the 217 patients, 127 were men and 90 were women. The mean age of the patients was 67 years (SD, 12 years; range, 30-95 years). No significant difference was observed in age, sex, pathological AJCC stages, surgical procedures, APR receipt or nonreceipt, and pathological tumor size between the two groups (Table 1). Moreover, all distal surgical margins in the study were free, and the mean margin distance from the distal edge of the tumor was $2.27 \mathrm{~cm}(\mathrm{SD}, 1.52 \mathrm{~cm})$. Distant metastasis was significantly higher in patients with advanced pathologic AJCC stages (stages II-III compared with stage I); those receiving adjuvant RT, $\mathrm{CT}$, or CCRT; and those with lower-third rectal cancers (Table 1). Specifically, the distant metastasis rates observed in patients with middle- and lower-third rectal cancers were $9.47 \%$ and $21.21 \%$, respectively, compared with those in patients with upper-third rectal cancers $(1.79 \%)$. The characteristics of patients with rectal adenocarcinoma at different tumor locations are presented in Table 2. The proportions of patients undergoing APR (100.00\%), adjuvant CCRT $(73.33 \%)$, and adjuvant RT $(50.00 \%)$ were significantly higher among those having tumors in the lower third rectum (Table 2). To examine prognostic factors for distant metastasis, we performed univariate and multivariate Cox regression analyses of the distant metastasis rate in patients with rectal adenocarcinoma (Table 3). After including only model variables of distant metastasis with the highest or lowest univariate risk, we observed that middle- and lower-third rectal cancers, pathological AJCC stage III, APR receipt, adjuvant RT receipt, and adjuvant $\mathrm{CT}$ receipt were poor prognostic factors. However, after the execution of a multivariate Cox regression analysis of the distant metastasis rate in patients with rectal adenocarcinoma, tumor locations and AJCC stages were identified as prognostic risk factors (Table 3). The adjusted HRs (aHRs) of distant metastasis for the upper-third, middle-third, and AJCC stage I-II rectal cancers were $0.08(95 \% \mathrm{CI}$, $0.01-0.69 ; p=0.021), 0.41$ (95\% CI, 0.15-0.99; $p=0.047)$, and 0.20 (95\% CI, $0.10-0.66 ; p=0.008)$, respectively.

Table 1. Characteristics of patients with rectal adenocarcinoma with and without distant metastasis.

\begin{tabular}{|c|c|c|c|}
\hline & $\begin{array}{l}\text { Distant metastasis, } n \\
(\%)\end{array}$ & $\begin{array}{l}\text { No metastasis, } n \\
(\%)\end{array}$ & $P$ value \\
\hline Sex & & & 1.000 \\
\hline Male & $13(10.24)$ & 114 (89.76) & \\
\hline Female & $11(12.22)$ & $79(87.78)$ & \\
\hline Age (years) & & & 0.190 \\
\hline$>65$ & $17(16.35)$ & $87(83.65)$ & \\
\hline$\leq 65$ & $7(6.19)$ & $106(93.81)$ & \\
\hline Pathological AJCC stage & & & $<0.0001$ \\
\hline I & $0(0.00)$ & $48(100)$ & \\
\hline II & $4(5.63)$ & 67 (94.37) & \\
\hline III & $20(20.41)$ & 78 (79.59) & \\
\hline Surgical procedure & & & 0.386 \\
\hline Open surgery & $22(12.29)$ & $157(87.71)$ & \\
\hline Laparoscopic surgery & $2(5.41)$ & 35 (94.59) & \\
\hline Pathological tumor size & & & 1.000 \\
\hline$\geq 5 \mathrm{~cm}$ & $4(10.81)$ & $33(89.19)$ & \\
\hline$<5 \mathrm{~cm}$ & $20(11.11)$ & $160(88.89)$ & \\
\hline Pathological T stage & & & 0.002 \\
\hline pT1 & $0(0.00)$ & $20(100)$ & \\
\hline pT2 & $1(1.69)$ & $58(98.31)$ & \\
\hline pT3 & $22(17.32)$ & $105(82.68)$ & \\
\hline pT4 & $1(9.09)$ & $10(90.91)$ & \\
\hline Pathological N stages & & & $<0.0001$ \\
\hline pNO & $5(3.97)$ & $121(96.03)$ & \\
\hline pN1 & $6(10.71)$ & $50(89.29)$ & \\
\hline $\mathrm{pN} 2$ & $13(37.14)$ & $22(62.86)$ & \\
\hline Adjuvant CCRT & & & 0.030 \\
\hline Yes & $7(23.33)$ & $23(76.67)$ & \\
\hline No & $17(9.94)$ & $154(90.06)$ & \\
\hline Adjuvant RT & & & 0.009 \\
\hline Yes & $11(27.50)$ & $39(72.50)$ & \\
\hline No & $13(7.78)$ & $154(92.22)$ & \\
\hline Adjuvant CT & & & 0.002 \\
\hline Yes & 19 (18.10) & $86(81.90)$ & \\
\hline No & $5(4.46)$ & 107 (95.54) & \\
\hline APR & & & 0.239 \\
\hline Yes & $4(21.05)$ & 15 (78.95) & \\
\hline No & $20(5.05)$ & 178 (94.95) & \\
\hline Tumor location & & & 0.001 \\
\hline Upper third & $1(1.79)$ & $55(98.21)$ & \\
\hline Middle third & $9(9.47)$ & $86(90.53)$ & \\
\hline Lower third & $14(21.21)$ & $52(78.79)$ & \\
\hline Total & 24 & 193 & \\
\hline
\end{tabular}

$\mathrm{RT}$, radiotherapy; $\mathrm{CT}$, chemotherapy; CCRT, concurrent chemoradiotherapy; APR, abdominal perineal resection; AJCC, American Joint Committee on Cancer. 
Table 2. Characteristics of patients with rectal adenocarcinoma at different locations.

\begin{tabular}{|c|c|c|c|c|}
\hline & $\begin{array}{l}\text { Upper third, } n \\
(\%)\end{array}$ & $\begin{array}{l}\text { Middle third, } n \\
(\%)\end{array}$ & $\begin{array}{l}\text { Lower third, } n \\
\text { (\%) }\end{array}$ & $P$ value \\
\hline Sex & & & & 0.292 \\
\hline Male & $29(23.97)$ & $61(48.03)$ & 37 (29.13) & \\
\hline Female & $27(30.00)$ & 34 (37.78) & $29(32.22)$ & \\
\hline Age (years) & & & & 0.649 \\
\hline$>65$ & $31(25.20)$ & $57(46.34)$ & 35 (28.46) & \\
\hline$\leq 65$ & $25(26.60)$ & 38 (40.43) & 31 (32.98) & \\
\hline Pathological AJ & CC stage & & & 0.594 \\
\hline I & $16(33.33)$ & $20(41.67)$ & $12(25.00)$ & \\
\hline II & $19(23.46)$ & $29(35.80)$ & $23(28.40)$ & \\
\hline III & $21(21.43)$ & $46(46.94)$ & 31 (31.63) & \\
\hline Adjuvant CCRT & & & & $<0.0001$ \\
\hline Yes & $1(3.33)$ & $8(26.67)$ & $21(73.33)$ & \\
\hline No & 55 (29.41) & $87(46.52)$ & $45(24.06)$ & \\
\hline Adjuvant RT & & & & 0.002 \\
\hline Yes & $7(14.00)$ & $18(36.00)$ & $25(50.00)$ & \\
\hline No & $49(29.34)$ & 77 (46.11) & 41 (24.55) & \\
\hline Adjuvant CT & & & & 0.753 \\
\hline Yes & $25(23.81)$ & $46(43.81)$ & $34(32.28)$ & \\
\hline No & $31(27.68)$ & 49 (43.75) & $32(28.57)$ & \\
\hline APR & & & & $<0.0001$ \\
\hline Yes & $0(0.00)$ & $0(0.00)$ & $19(100)$ & \\
\hline No & $56(28.28)$ & $95(47.98)$ & 47 (23.74) & \\
\hline
\end{tabular}

Table 3. Cox proportional hazards model for the risk of distant metastasis among patients with rectal adenocarcinoma.

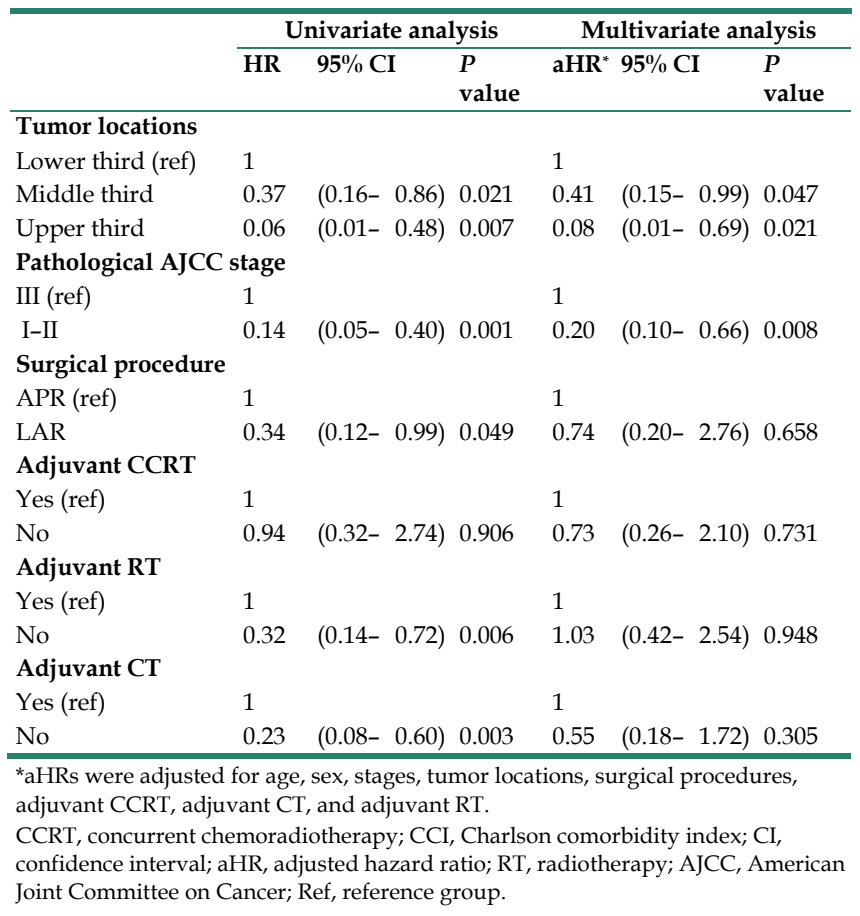

The 5-year distant metastasis rates among patients with rectal cancers at different locations were $22.71 \%$ (lower third), $12.47 \%$ (middle third), and $1.82 \%$ (upper third; Supplemental Table 1). The cumulative curve of distant metastasis among patients with tumors in the lower third rectum was steep within the first 5 years after surgery, almost reached a plateau after 6.25 years, and remained unchanged after 10 years (Figure 1). We also evaluated the metastatic sites by different locations of rectal cancers by using ANOVA (Table 4). A statistically significant trend of lung metastasis was observed in lower-third rectal cancer $(p=0.008)$, but no statistically significant trend of liver metastasis was observed in different locations of rectal cancers. The 5-year lung distant metastasis rate in lower-third rectal adenocarcinoma was also significantly high, with a metastasis rate of $13.33 \%$ in the lungs $(P=$ 0.001; Supplemental Table 2). In addition, the different metastatic sites at different locations of rectal adenocarcinoma were determined using the Kaplan-Meier method, and Kaplan-Meier survival curves were compared using the log-rank test (Figures 2 and 3). The 5-year lung metastasis rates among patients with different tumor locations such as the upper, middle, and lower third rectum were $0 \%$, $3.37 \%$, and $13.33 \%$, respectively (log-rank, $p=0.001$ ), and the 5-year liver metastasis rates among patients with upper-, middle-, and lower-third rectal cancers were $2.12 \%, 9.10 \%$, and $11.76 \%$, respectively (logrank, $p=0.096$; Figure 2). The 5-year OS rates were also very different with different locations of rectal adenocarcinoma. . The 5-year OS rates for upper, middle, and lower rectal cancers were $96 \%, 86 \%$, and $64 \%$, respectively (log-rank, $p<0.001$; Figure 4 ).

Table 4. Different metastatic sites at different locations of rectal adenocarcinoma.

\begin{tabular}{lllll}
\hline & $\begin{array}{l}\text { Upper third, } \boldsymbol{n} \\
(\mathbf{\%})\end{array}$ & $\begin{array}{l}\text { Middle third, } \boldsymbol{n} \text { Lower third, } \boldsymbol{n} \\
\mathbf{( \% )}\end{array}$ & $\boldsymbol{P}$ value \\
$\mathbf{( \% )}$
\end{tabular}

\section{Discussion}

Patients with rectal adenocarcinoma receiving TME have a local recurrence of approximately $4 \%-10 \%,[7,14]$ but they still have a high distant metastasis rate. $[15,16]$ The most common metastatic sites are the liver and lungs.[17] The metastasis could shorten the life spans of patients with rectal adenocarcinoma, and retreatment such as salvage surgery, CT, or RT might be necessary.[18] When patients with rectal adenocarcinoma receiving TME developed distant metastasis, the 5-year OS was dismal.[16] Till now, no clear predictive risk factor for distant metastasis in patients with rectal adenocarcinoma receiving TME is available. Data 
elucidating the patterns of distant metastasis, including the required duration of follow-up, whether the metastasis rate could reach a plateau, the most common metastatic sites (the liver or lungs), and whether a close monitoring of the liver, lung, or both metastases is sufficient, are limited.

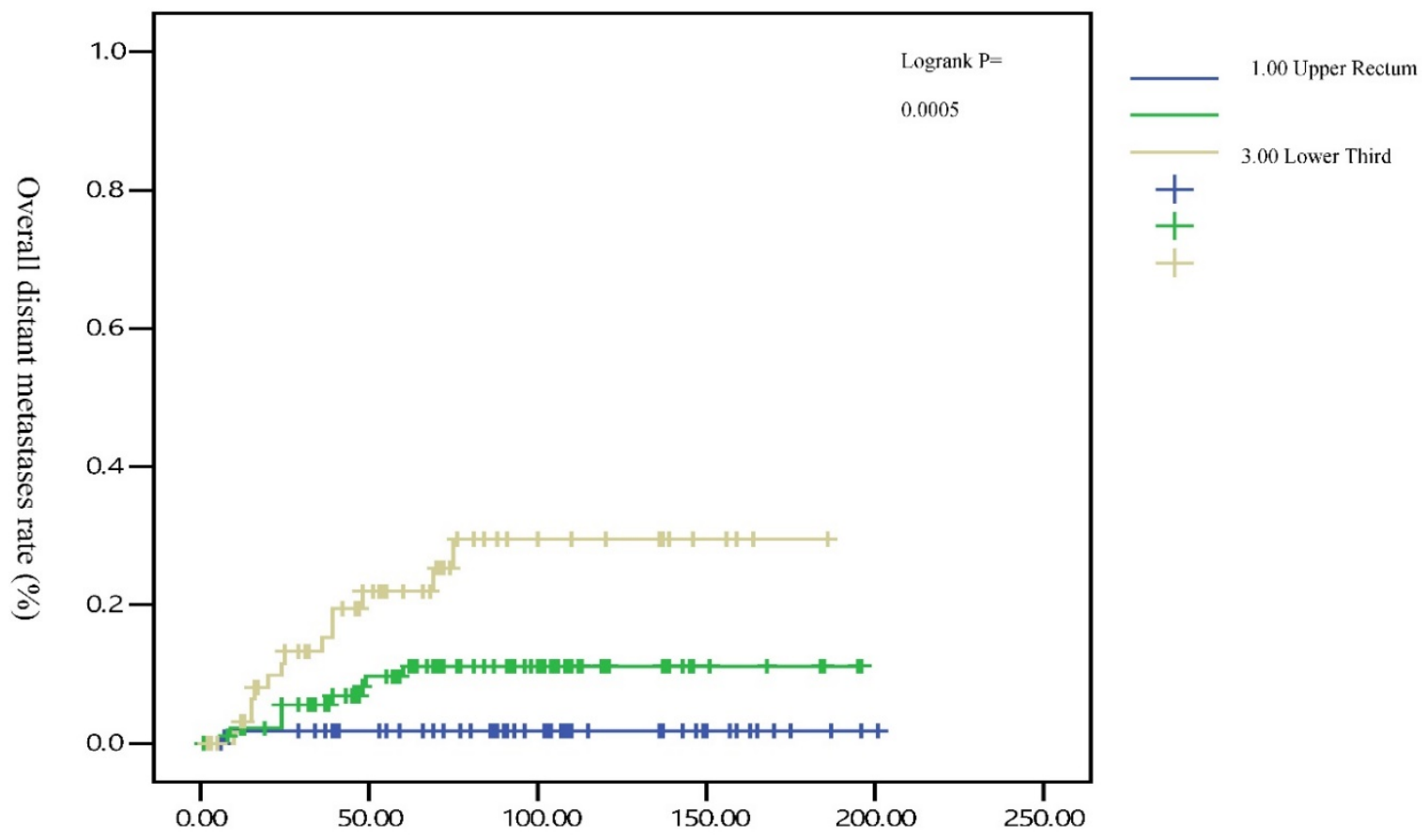

Time after operation (months)

Figure 1. Five-year overall distant metastasis rates by different locations of rectal adenocarcinoma

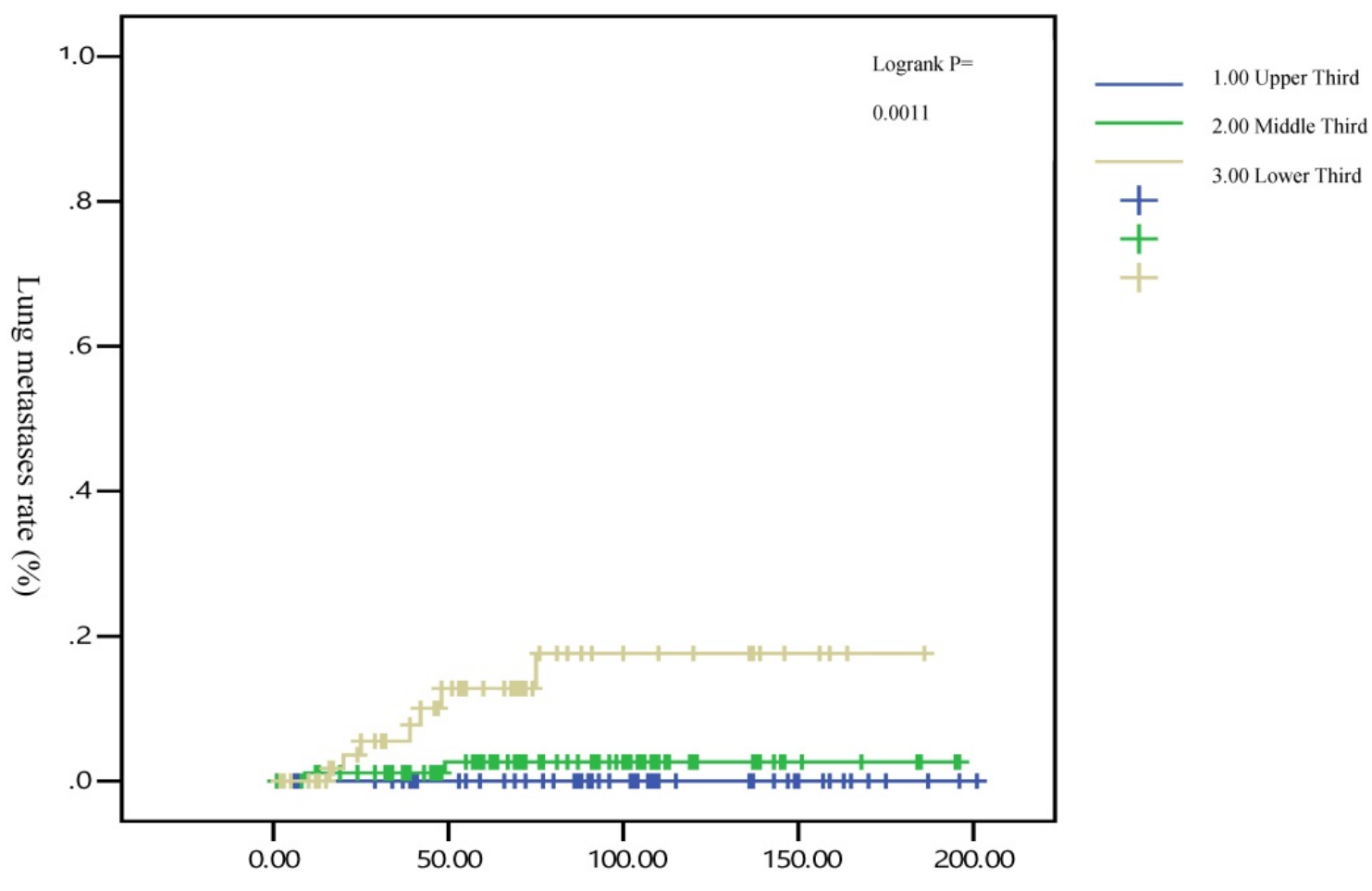

Time after operation (months)

Figure 2. Five-year lung metastasis rates by different locations of rectal adenocarcinoma 


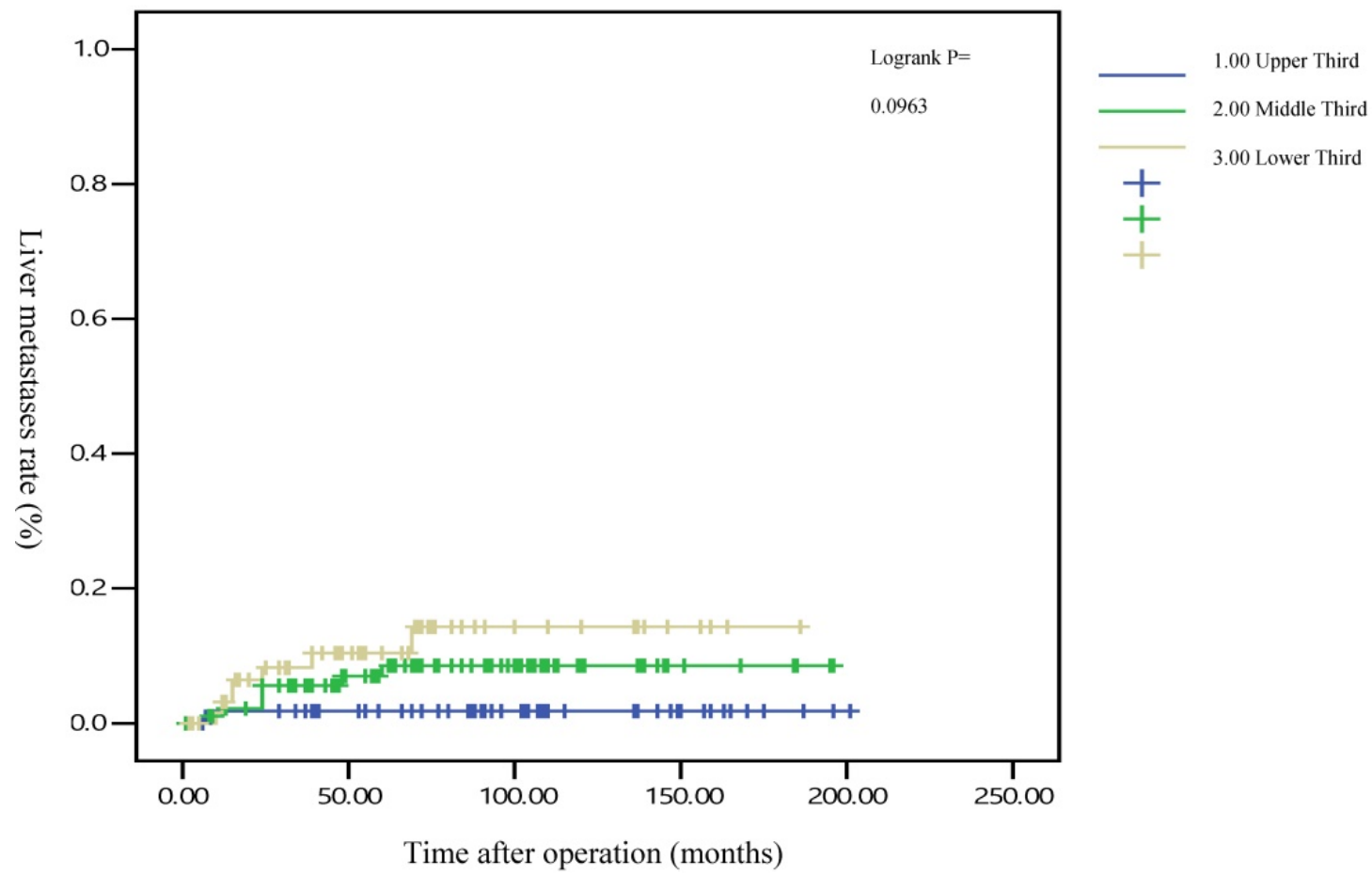

Figure 3. Five-year liver metastasis rates by different locations of rectal adenocarcinoma

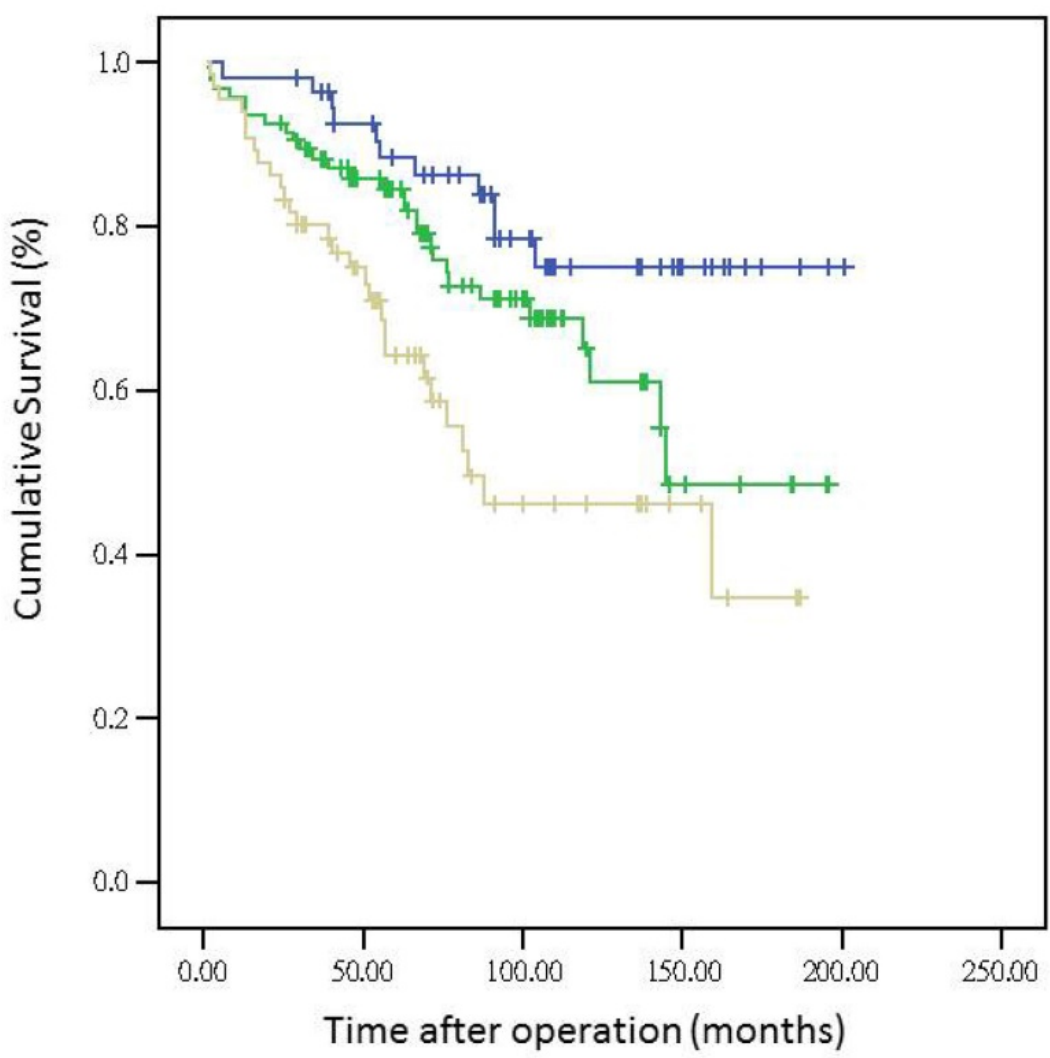

$$
\begin{aligned}
& \text { Location } \\
& \begin{aligned}
- & 1.00 \text { Upper Rectum } \\
\hline & 2.00 \text { Middle Rectum } \\
& 3.00 \text { Low Rectum } \\
+\quad & 1.00 \text { censored } \\
+\quad & 2.00 \text { censored } \\
+\quad & 3.00 \text { censored }
\end{aligned}
\end{aligned}
$$

Figure 4. Five-year overall survival rates by different locations of rectal adenocarcinoma

The metastasis rate was higher in advanced $\mathrm{pT}$, $\mathrm{pN}$, and pathologic AJCC stages (Table 1). Our findings are comparable to those of previous studies[19-21]; nevertheless, although these predictive factors were used for determining colon and rectal cancers, they were not specific to rectal adenocarcinoma. However, the metastasis rate remained high in patients receiving adjuvant $\mathrm{RT}, \mathrm{CT}$, and CCRT in our study (Table 1), which is inconsistent with the findings of previous studies[16, 22, 23]; these results 
might be masked by adverse pathologic risk factors such as close margin, perineural invasion, lymphatic vascular invasion, advanced pathologic AJCC stages, and surgeons' concerns during operation. A multivariate Cox regression analysis revealed that these findings were not statistically significant (Table 3). Another notable finding was the association between tumor locations and distant metastasis rates (Table 1). We separated different locations of rectal adenocarcinoma in our study, and no statistically significant difference was observed in sex, pathologic AJCC stages, and age between the upper-, middle-, and lower-third rectal adenocarcinomas (Table 2). Statistical significance was noted in adjuvant RT, adjuvant CCRT, and APR receipt. These findings indicate that more patients with lower-third rectal adenocarcinoma received adjuvant RT, adjuvant CCRT, and APR, which might be because of the difficulties in surgical approach due to anatomical structures[3, 24]; however, no statistical significance in pathologic AJCC stages was observed between the upper-, middle-, and lower-third rectal adenocarcinomas (Table 2). In the current study, all distal surgical margins were free, even in lower-third rectal adenocarcinoma, because we performed APR rather than the sphincter-sparing procedure, leading to positive margins. Therefore, more patients with lower-third rectal cancer received adjuvant RT and adjuvant CCRT; adjuvant RT or adjuvant CCRT exhibited more favorable outcomes in patients with rectal cancer when compared with nonadjuvant treatments.[22, 23, 25, 26] The current study findings demonstrate that although the number of patients with middle- and lower-third rectal adenocarcinomas receiving adjuvant CCRT or RT was higher, the distant metastasis rate remained higher in middleand lower-third rectal cancers. According to our review of the relevant literature, this is the first study to demonstrate that the distant metastasis rate and metastatic sites differed with different locations of rectal adenocarcinoma. In addition, the OS rate was influenced by the different locations of rectal cancer. A trend of higher distant metastasis and mortality rates was observed in patients with middle and lower rectal adenocarcinoma compared with those with upper rectal adenocarcinoma (Figures 1 and 4).

After the execution of a multivariate Cox regression analysis of the distant metastasis rate in patients with rectal adenocarcinoma, tumor locations and AJCC stages were identified as prognostic risk factors (Table 3). Although the distributions of pathological stages between the upper-, middle-, and lower-third rectal adenocarcinomas were balanced (Table 2), the location of the rectal adenocarcinoma was the independent predictive factor for distant metastasis (Table 3). The aHRs of distant metastasis for the upper- and middle-third rectal cancers were 0.08 (95\% CI, 0.01-0.69; $P=0.021)$ and 0.41 (95\% CI, 0.15-0.99; $P=0.047)$, respectively. The locations of rectal adenocarcinoma were closer to the anal verge, and the higher distant metastasis rates were significant (Figure 1). Rectal adenocarcinoma can spread through lymphatic and hematogenous dissemination, as well as through contiguous and transperitoneal routes.[27-29] The most common metastatic sites are the liver and lungs.[30, 31] Because the venous drainage of the intestinal tract passes through the portal system, the first site of hematogenous dissemination is usually the liver, followed by the lungs.[27, 31, 32] In addition, tumors arising from the distal rectum may initially metastasize to the lungs because the inferior rectal vein drains into the inferior vena cava rather than into the portal venous system.[6, 33, 34] Our clinical finding is compatible with previous theories. $[6,33,34]$ A statistically significant trend of lung metastasis was observed in lower-third rectal cancers $(p=0.008)$, but no statistically significant trend of liver metastasis was observed in different locations of rectal cancers (Table 4). The 5-year lung distant metastasis rate in lower-third rectal adenocarcinoma was also significantly high, with a metastasis rate of $13.33 \%$ in the lungs $(p=0.001$; Supplemental Table 2). The 5 -year lung metastasis rates among patients with tumors at different locations such as the upper, middle, and lower third rectum were $0 \%, 3.37 \%$, and $13.33 \%$, respectively (log-rank, $p=0.001$ ), and the 5 -year liver metastasis rates among patients with upper-, middle-, and lower-third rectal cancers were $2.12 \%, 9.10 \%$, and $11.76 \%$, respectively (log-rank, $p=$ 0.096) (Figure 2). This is the first clinical study to prove that lower-third rectal cancer has an equal metastatic trend toward the lungs and liver, whereas upper- and middle-third rectal cancers have a strong metastatic trend toward the liver (Table 4).

In all, 14 patients with lower-third rectal adenocarcinoma exhibited distant metastasis and 7 patients exhibited lung metastasis first rather than liver metastasis. Our results suggest that routine surveillance should include lung survey, particularly in lower-third rectal adenocarcinoma.

Several meta-analyses have supported a modest but significant survival benefit from an intensive surveillance strategy after resection of a colorectal cancer.[35-39] Our results reveal that no more distant metastasis occurred after 5 years in patients with upper and middle rectal cancers, and the distant metastasis might reach a plateau after 5 years (Figure 1). However, metastasis was still observed after 5 years in patients with lower-third rectal cancer, and a 
metastatic plateau seemed to be reached after 6.25 years. These findings imply that annual surveillance of the chest, abdomen, and pelvis should be performed for at least 6.25 years for lower-third rectal cancers (Figure 1). After 5 years, the metastatic site in lower-third rectal cancer was the lungs instead of the liver (Figures 2 and 3). Therefore, chest surveillance should be performed for more than 6 years in patients with lower-third rectal cancer (Figure 2).

No clinical data are available to prove that patients with lower-third rectal cancer receiving TME still have an equal distant metastasis trend toward the lungs and liver, even after 6 years. Our data demonstrate the clinical findings in patients with rectal cancer receiving TME. Furthermore, this is the first study to demonstrate a poor OS rate and high metastasis rate in distal rectal adenocarcinoma, irrespective of adjuvant treatments such as CT, RT, or CCRT. Longer intensive surveillance of the chest, abdomen, and pelvis could be necessary after TME in distal rectal adenocarcinoma.

\section{Conclusions}

A poor OS rate and high lung or liver metastasis rate were observed in distal rectal adenocarcinoma. Therefore, longer intensive surveillance of the chest, abdomen, and pelvis could be necessary after TME in distal rectal adenocarcinoma.

\section{Abbreviations}

RT: radiotherapy; CT: chemotherapy; CCRT: concurrent chemoradiotherapy; LAR: low anterior resection; TME: total mesorectal excision; AJCC: American Joint Committee on Cancer; CI: confidence interval; aHR: adjusted hazard ratio; Ref: reference group; OS: Overall survival.

\section{Supplementary Material}

Supplementary tables.

http://www.jcancer.org/v09p0950s1.pdf

\section{Acknowledgement}

Szu-Yuan Wu's work is supported by Taipei Medical University (TMU105-AE1-B26) \&Wan Fang Hospital funding 107-wf-swf-08.

\section{Competing Interests}

The authors have declared that no competing interest exists.

\section{References}

1. National Health Insurance Administration, Ministry of Health and Welfare, Taiwan, R.O.C. National Health Insurance Annual Report 2014-2015. 2016.

2. Northover J, Glynne-Jones R, Sebag-Montefiore D, James R, Meadows H, Wan $S$, et al. Chemoradiation for the treatment of epidermoid anal cancer: 13-year follow-up of the first randomised UKCCCR Anal Cancer Trial (ACT I). British journal of cancer. 2010; 102: 1123-8.
3. Chen CH, Wei PL, Hsieh MC, Lin EK, Chiou JF, Lu YJ, et al. The outcomes of therapeutic decision in lower 3 rd rectal cancer patients. Medicine (Baltimore). 2016; 95: e4638.

4. Heald RJ, Ryall RD. Recurrence and survival after total mesorectal excision for rectal cancer. Lancet. 1986; 1: 1479-82.

5. Heald RJ. Total mesorectal excision is optimal surgery for rectal cancer: a Scandinavian consensus. The British journal of surgery. 1995; 82: 1297-9.

6. Heald RJ, Husband EM, Ryall RD. The mesorectum in rectal cancer surgery--the clue to pelvic recurrence? The British journal of surgery. 1982; 69: 613-6.

7. Heald RJ, Moran BJ, Ryall RD, Sexton R, MacFarlane JK. Rectal cancer: the Basingstoke experience of total mesorectal excision, 1978-1997. Arch Surg. 1998; 133: 894-9.

8. Heald RJ, Moran BJ, Brown G, Daniels IR. Optimal total mesorectal excision for rectal cancer is by dissection in front of Denonvilliers' fascia. The British journal of surgery. 2004; 91: 121-3.

9. Edge S, Byrd D, Compton C. AJCC (American Joint Committee on Cancer) Cancer Staging Manual. 7th ed. New York: Springer; 2010.

10. Tominaga T, Sakabe T, Koyama Y, Hamano K, Yasutomi M, Takahashi T, et al. Prognostic factors for patients with colon or rectal carcinoma treated with resection only. Five-year follow-up report. Cancer. 1996; 78: 403-8.

11. Shepherd NA, Baxter KJ, Love SB. The prognostic importance of peritoneal involvement in colonic cancer: a prospective evaluation. Gastroenterology. 1997; 112: 1096-102.

12. Zeng Z, Cohen AM, Hajdu S, Sternberg SS, Sigurdson ER, Enker W. Serosal cytologic study to determine free mesothelial penetration of intraperitoneal colon cancer. Cancer. 1992; 70: 737-40.

13. Chien-Hsin Chen, Ping-Kun Hsiao, En-Kwang Lin, Yen-Jung Lu, Wu S-Y. A critical reappraisal for the value of tumor size as a prognostic variable in rectal adenocarcinoma. Journal of Cancer. 2017; 8(10): 1927-1934.

14. Enker WE, Thaler HT, Cranor ML, Polyak T. Total mesorectal excision in the operative treatment of carcinoma of the rectum. Journal of the American College of Surgeons. 1995; 181: 335-46.

15. Russell AH, Harris J, Rosenberg PJ, Sause WT, Fisher BJ, Hoffman JP, et al. Anal sphincter conservation for patients with adenocarcinoma of the distal rectum: long-term results of radiation therapy oncology group protocol 89-02. International journal of radiation oncology, biology, physics. 2000; 46: 313-22.

16. Tiselius C, Gunnarsson U, Smedh K, Glimelius B, Pahlman L. Patients with rectal cancer receiving adjuvant chemotherapy have an increased survival: a population-based longitudinal study. Annals of oncology : official journal of the European Society for Medical Oncology / ESMO. 2013; 24: 160-5.

17. Gomez D, Sangha VK, Morris-Stiff G, Malik HZ, Guthrie AJ, Toogood GJ, et al. Outcomes of intensive surveillance after resection of hepatic colorectal metastases. The British journal of surgery. 2010; 97: 1552-60.

18. Robertson DJ, Stukel TA, Gottlieb DJ, Sutherland JM, Fisher ES. Survival after hepatic resection of colorectal cancer metastases: a national experience. Cancer. 2009; 115: 752-9.

19. Gunderson LL, Sargent DJ, Tepper JE, O'Connell MJ, Allmer C, Smalley SR, et al. Impact of $\mathrm{T}$ and $\mathrm{N}$ substage on survival and disease relapse in adjuvant rectal cancer: a pooled analysis. International journal of radiation oncology, biology, physics. 2002; 54: 386-96.

20. Gunderson LL, Sargent DJ, Tepper JE, Wolmark N, O'Connell MJ, Begovic M, et al. Impact of $\mathrm{T}$ and $\mathrm{N}$ stage and treatment on survival and relapse in adjuvant rectal cancer: a pooled analysis. Journal of clinical oncology : official journal of the American Society of Clinical Oncology. 2004; 22: 1785-96.

21. Gunderson LL, Callister M, Marschke R, Young-Fadok T, Heppell J, Efron J. Stratification of rectal cancer stage for selection of postoperative chemoradiotherapy: current status. Gastrointestinal cancer research : GCR. 2008; 2 : 25-33.

22. Adjuvant radiotherapy for rectal cancer: a systematic overview of 8,507 patients from 22 randomised trials. Lancet. 2001; 358: 1291-304.

23. Tepper JE, O'Connell $\mathrm{M}$, Niedzwiecki $\mathrm{D}$, Hollis DR, Benson AB, 3rd, Cummings $\mathrm{B}$, et al. Adjuvant therapy in rectal cancer: analysis of stage, sex, and local control--final report of intergroup 0114. Journal of clinical oncology : official journal of the American Society of Clinical Oncology. 2002; 20: 1744-50.

24. Bordeianou L, Maguire LH, Alavi K, Sudan R, Wise PE, Kaiser AM. Sphincter-sparing surgery in patients with low-lying rectal cancer: techniques, oncologic outcomes, and functional results. Journal of gastrointestinal surgery : official journal of the Society for Surgery of the Alimentary Tract. 2014; 18: 1358-72.

25. Holyoke ED, Mittelman A, et al. Prolongation of the disease-free interval in surgically treated rectal carcinoma. The New England journal of medicine. 1985; 312: 1465-72.

26. Krook JE, Moertel CG, Gunderson LL, Wieand HS, Collins RT, Beart RW, et al. Effective surgical adjuvant therapy for high-risk rectal carcinoma. The New England journal of medicine. 1991; 324: 709-15.

27. Chambers AF, Groom AC, MacDonald IC. Dissemination and growth of cancer cells in metastatic sites. Nature reviews Cancer. 2002; 2: 563-72.

28. Weiss L, Grundmann E, Torhorst J, Hartveit F, Moberg I, Eder M, et al. Haematogenous metastatic patterns in colonic carcinoma: an analysis of 1541 necropsies. The Journal of pathology. 1986; 150: 195-203.

29. Schimanski CC, Schwald S, Simiantonaki N, Jayasinghe C, Gonner U, Wilsberg V, et al. Effect of chemokine receptors CXCR4 and CCR7 on the metastatic behavior of human colorectal cancer. Clinical cancer research : an 
official journal of the American Association for Cancer Research. 2005; 11: 1743-50.

30. Kabbinavar F, Hurwitz HI, Fehrenbacher L, Meropol NJ, Novotny WF, Lieberman G, et al. Phase II, randomized trial comparing bevacizumab plus fluorouracil (FU)/leucovorin (LV) with FU/LV alone in patients with metastatic colorectal cancer. Journal of clinical oncology : official journal of the American Society of Clinical Oncology. 2003; 21: 60-5.

31. Riihimaki M, Hemminki A, Sundquist J, Hemminki K. Patterns of metastasis in colon and rectal cancer. Scientific reports. 2016; 6: 29765.

32. Fortner JG, Silva JS, Golbey RB, Cox EB, Maclean BJ. Multivariate analysis of a personal series of 247 consecutive patients with liver metastases from colorectal cancer. I. Treatment by hepatic resection. Annals of surgery. 1984; 199: 306-16.

33. Nelson H, Petrelli N, Carlin A, Couture J, Fleshman J, Guillem J, et al. Guidelines 2000 for colon and rectal cancer surgery. Journal of the National Cancer Institute. 2001; 93: 583-96.

34. Moriya Y, Akasu T, Fujita S, Yamamoto S. Total pelvic exenteration with distal sacrectomy for fixed recurrent rectal cancer in the pelvis. Diseases of the colon and rectum. 2004; 47: 2047-53; discussion 53-4.

35. Mokhles S, Macbeth F, Farewell V, Fiorentino F, Williams NR, Younes RN, et al. Meta-analysis of colorectal cancer follow-up after potentially curative resection. The British journal of surgery. 2016; 103: 1259-68.

36. Tjandra JJ, Chan MK. Follow-up after curative resection of colorectal cancer: a meta-analysis. Diseases of the colon and rectum. 2007; 50: 1783-99.

37. Renehan AG, Egger M, Saunders MP, O'Dwyer ST. Impact on survival of intensive follow up after curative resection for colorectal cancer: systematic review and meta-analysis of randomised trials. Bmj. 2002; 324: 813.

38. Desch CE, Benson AB, 3rd, Somerfield MR, Flynn PJ, Krause C, Loprinzi CL, et al. Colorectal cancer surveillance: 2005 update of an American Society of Clinical Oncology practice guideline. Journal of clinical oncology : official journal of the American Society of Clinical Oncology. 2005; 23: 8512-9.

39. Scheer A, Auer RA. Surveillance after curative resection of colorectal cancer. Clinics in colon and rectal surgery. 2009; 22: 242-50. 